\title{
Demagnetizer Device
}

National Cancer Institute

\section{Source}

National Cancer Institute. Demagnetizer Device. NCI Thesaurus. Code C49914.

A device designed to remove the magnetic properties of an object or material. 